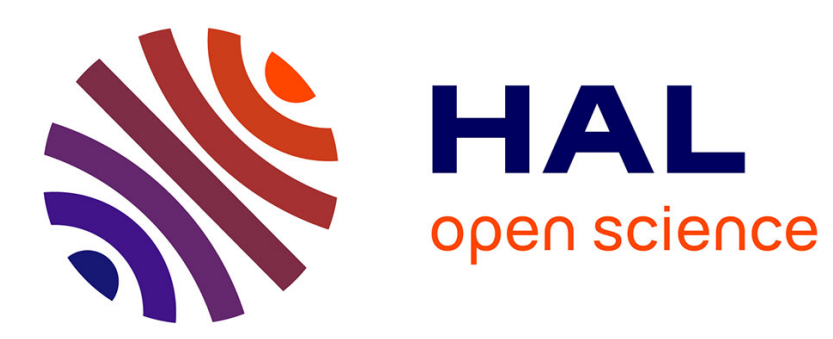

\title{
La complexité médicale appliquée à l'allergologie : causes et solutions
}

M. Demoly, J.L. Bourrain, P. Demoly

\section{To cite this version:}

M. Demoly, J.L. Bourrain, P. Demoly. La complexité médicale appliquée à l'allergologie: causes et solutions. Revue francaise d'allergologie, 2019, 59, pp.336 - 340. 10.1016/j.reval.2019.01.007 . hal-03484918

\section{HAL Id: hal-03484918 \\ https://hal.science/hal-03484918}

Submitted on 21 Dec 2021

HAL is a multi-disciplinary open access archive for the deposit and dissemination of scientific research documents, whether they are published or not. The documents may come from teaching and research institutions in France or abroad, or from public or private research centers.
L'archive ouverte pluridisciplinaire HAL, est destinée au dépôt et à la diffusion de documents scientifiques de niveau recherche, publiés ou non, émanant des établissements d'enseignement et de recherche français ou étrangers, des laboratoires publics ou privés.

\section{다)(1) $(5$}

Distributed under a Creative Commons Attribution - NonCommerciall 4.0 International 
Version of Record: https://www.sciencedirect.com/science/article/pii/S1877032019300132

Manuscript_0f9656dbc0817d0c467ae4a6b46dad37

La complexité médicale appliquée à l'allergologie : causes et solutions

\section{Medical complexity relating to allergology:} causes and solutions

\section{Maxime Demoly ${ }^{1}$, Jean Luc Bourrain, Pascal Demoly}

Exploration des Allergies, Département de Pneumologie et Addictologie, Hôpital Arnaud de Villeneuve, CHU de Montpellier, France.

${ }^{1}$ Porteur du projet de société AdviceMedica (http:www.advicemedica.com)

Auteur correspondant :

Pr Pascal DEMOLY

Département de Pneumologie et Addictologie - Hôpital Arnaud de Villeneuve

CHU de Montpellier, 34295 Montpellier cedex 05

Tel: 04673361 17, Fax 0467996429

Mail: pascal.demoly@inserm.fr 


\title{
RESUME
}

Complexité et incertitude médicales sont source de retards diagnostiques et de mauvaises stratégies thérapeutiques et donc d'augmentation des coûts et des erreurs médicales. Leurs mécanismes sont multiples et liés aussi bien au patient et à sa pathologie qu'aux modes de prises en charge. Parmi les nombreuses solutions pour y faire face, l'utilisation de bases de données dynamiques et la collaboration entre professionnels sont particulièrement appréciées par les praticiens. C'est ce que nous avons mis en place avec la plateforme collaborative AdviceMedica, développée initialement sur un mode associatif et académique. Elle est parfaitement fonctionnelle et utilisée quotidiennement. Elle utilise depuis peu les outils d'analyse du langage naturel. L'allergologie devient la première spécialité en France à disposer d'un réseau d'entre aide collaborative, bientôt renforcé par des analyses automatiques à la pointe de l'intelligence artificielle.

Mots clés: analyse du langage naturel, allergologie, erreur médicale, intelligence artificielle, intelligence collective, plateforme collaborative

\begin{abstract}
Medical complexity and uncertainty constitute a source of diagnostic delays and poor therapeutic strategies, and thus of increased costs and medical errors. Multiple mechanisms are involved and are related to patients and their pathology as well as to treatment methods. Among the many possible solutions, use of dynamic databases and collaboration between professionals are particularly appreciated by practitioners. This is what we have put in place with the AdviceMedica consultative platform, which was initially developed in an associative and academic way. It is perfectly functional and is used daily. It has recently begun to utilise natural language processing tools. Allergology is the first specialty in France to have a network of collaborative help, which will soon be further strengthened by automated analysis methods at the very forefront of artificial intelligence.
\end{abstract}

Keywords: allergology, medical error, artificial intelligence, collective intelligence, collaborative platform, natural language analysis

\section{COMPLEXITE, INCERTITUDE ET EXPERTISE MEDICALES}

\section{Définition et conséquences}

Le diagnostic et la prise en charge des maladies suivent des règles, apprises à la faculté et mises à jour continuellement au cours de la pratique professionnelle. L'enseignement facultaire des maladies s'articule autour de cours théoriques (les premières années, par l'étude des grands mécanismes physiopathologiques et leurs dysfonctionnements) et de l'analyse de cas typiques (ou vignettes ou illness scripts) $(1,2)$. Par la suite, des connaissances plus organisées et une compréhension plus fine 
de chaque pathologie viennent avec l'expérience clinique individuelle, le nombre de cas de patients vus par le praticien pour une pathologie donnée, et leur diversité par rapport à ce profil de patient moyen (les variantes pour une même maladie constituant ce qu'on appelle les instances du script). Le diagnostic dans la vraie vie, pas toujours simple, répond à une confrontation entre les symptômes (histoire clinique) et signes du patient, le contexte médico-psycho-social, les résultats de quelques examens complémentaires et la connaissance théorique et pratique que nous en avons, permettant de classer le patient dans telle ou telle catégorie de maladies (approche probabiliste) (2). Nous devons poser notre raisonnement et nos décisions dans l'hypothèse que le patient est dans la moyenne, et devons gérer l'éventualité où le patient dévie de cette moyenne $(2,3)$, ce qui a fait dire à Hippocrate (4): "La vie est courte, l'art est long, l'occasion fugitive, l'expérience trompeuse, le jugement difficile ».

Les décisions de soins doivent ensuite être fondées sur des schémas de prise en charge élaborés a priori et scientifiquement valides. Si cela est plus facile avec l'expérience, le médecin aura tout le temps des patients singuliers. Ainsi, nos décisions peuvent varier selon les circonstances, et d'un patient à l'autre avec les mêmes circonstances ; c'est ce que soulignent les dernières avancées en matière de médecine basée sur les preuves (5); cette dernière se définissant comme l'utilisation consciencieuse, explicite et judicieuse des meilleures données disponibles pour la prise de décisions concernant les soins à prodiguer à chaque patient. Elle complète la médecine personnalisée ou médecine 4P (personnalisée, préventive, prédictive et participative), récemment rebaptisée médecine 5P (pour médecine pertinente, des preuves).

Deux formes d'incertitudes médicales se dégagent alors (3) : l'une résultant d'une maîtrise incomplète ou imparfaite de la connaissance médicale et l'autre découlant des limites propres à la science médicale actuelle et au contexte institutionnel.

La complexité médicale est parfois source de retard diagnostics et de mauvaises stratégies thérapeutiques et donc d'augmentation des coûts et des erreurs médicales. Ces erreurs procèdent de multiples mécanismes et peuvent être liées aussi bien au patient, ses conditions de vie, son état psychologique, sa pathologie (6), qu'aux médecins et modes de prises en charge (7). L'erreur médicale (diagnostique ou thérapeutique) par défaut de connaissances et le non recours à un tiers est considérée par le législateur comme une perte de chance pour le patient et est condamnable juridiquement. Les données de la littérature à ce sujet sont rares et floues; on avance en France jusqu'à 450000 évènements indésirables médicamenteux graves et 50000 morts par an pour un coût annuel proche du demi-milliard d'euros (8). Selon la MACSF, principal assureur de médecins, les 6 spécialités les plus concernées par les plaintes sont, dans l'ordre : la chirurgie orthopédique, la chirurgie du rachis, la médecine générale, l'anesthésie-réanimation, l'ophtalmologie et la radiologie.

\section{Solutions}

Les solutions proposées pour faire face à la complexité et à l'incertitude médicales regroupent la formation (initiale et continue), l'optimisation du parcours de soins avec une prise en charge en réseau, l'implémentation de protocoles et guides de diagnostic et de traitement et la collaboration entre professionnels. 
Une approche plus collective de la médecine a gagné en légitimité ces dernières années, par rapport à la figure traditionnelle du médecin décidant seul de façon autonome et accordant une primauté à son expérience personnelle (1). Celle-ci consiste à privilégier le recours aux recommandations pour la pratique clinique (RPC) et à l'avis des pairs pour prendre des décisions. Les RPC, quand elles existent, concernent le plus souvent le "patient moyen " alors que, une fois de plus, notre expérience quotidienne concerne le « patient déviant ».

La collaboration entre professionnels est particulièrement appréciée par les praticiens ; elle prend en compte la spécificité d'un patient particulier. Elle est prévue par deux articles du code de santé publique, repris dans le Code de Déontologie Médicale du Conseil National de l'Ordre des Médecins (Tableau 1). Elle est la base des réseaux de soins et de la médecine à trois niveaux (généralistes, spécialistes, hyper/sur-spécialistes). Elle est obligatoire dans le cadre des réunions de concertation pluridisciplinaire (RCP) en cancérologie par exemple, depuis le premier plan cancer en 2000 (9). Elle pourrait le devenir dans d'autres domaines, notamment en pathologies respiratoires sévères (pour la prescription des biothérapies coûteuses après élimination des diagnostics différentiels par exemple).

\section{Analyse collective des cas complexes}

L'analyse collective des cas patients est de plus en plus pratique courante. Les médecins y trouvent un réel intérêt pour leur propre pratique. Elle met en avant la notion d'expertise et de centres / personnes de références. Mais qu'est-ce que l'expertise?

L'expertise a été étudiée par les sociologues médicaux $(1,3,6,7,9)$ et est associée à une meilleure structure et représentation mentale des maladies, des cas patients et à une capacité à reconnaître les cas éloignés de la norme (1). Elle est donc liée au nombre d'instances vues par script, à la capacité de mémorisation de chaque praticien et à la capacité d'établir des similarités entre le cas présent éloigné de la norme et des cas passés. L'expert a déjà vu des cas similaires et les a mémorisés. Les experts peuvent cependant commettre des erreurs, particulièrement s'ils ne font pas assez attention aux détails et prennent des raccourcis (1), d'où l'intérêt d'une analyse collective.

L'analyse de bases de données dynamiques telles qu'Allerdata ${ }^{\circledR}$ (http://allerdata.com/) pour la gestion des résultats d'IgE moléculaires est une aide précieuse également. Développée par des médecins allergologues et piloté par le Dr Hervé Masson, cette application assure non seulement une veille scientifique dans ce domaine mais aussi fournit un petit algorithme d'information / décision vis à vis des allergènes croisant ou pas. Telle est également le cas pour la base de données EASSAFE ${ }^{\circledR}$ (http://www.eassafe.com/) pilotée par le Dr Jean-François Fontaine, en ce qui concerne la composition des produits agro-alimentaires, cosmétiques et chimiques.

\section{VERS UNE MEDECINE INTELLIGENTE}

\section{Intelligence artificielle, données volumineuses de santé et pratique médicale}

Le monde de la santé est sûrement I'un des secteurs où les enjeux de l'intelligence artificielle (IA) sont les plus importants (10). Depuis la définition restrictive en 1956 par John McCarthy comme « la science et l'ingénierie de la fabrication de machines intelligentes ", I'IA est devenue un pan complet de recherche regroupant plusieurs technologies basées sur des algorithmes mathématiques 
appliqués à de nombreuses méthodes dont l'analyse profonde ou data mining, l'apprentissage automatique par renforcement ou machine learning, l'analyse du langage naturel ou natural language processing, les réseaux neuronaux ou neuronal networks et a bénéficié de progrès considérables à partir des années 2010, grâce à l'arrivée de nouveaux algorithmes, de la mise à disposition de volumineuses bases de données (Big data) et l'augmentation des puissances de calcul. Face à la suprématie des Etats-Unis et de la Chine dans ce domaine, la France et l'Europe ne doivent pas baisser les bras, nous rappelle le rapport du Pr Cédric Villani (10).

La France a été visionnaire avec la mise en place il y a vingt ans du Système national d'information inter-régimes de l'assurance maladie (Sniiram) et son chaînage au programme de médicalisation des systèmes d'information (PMSI). II couvre 99\% de la population. La loi du 27 janvier 2016, consacrée à la modernisation de ce système, traite de la création " d'un accès ouvert aux données de santé » (11) et créé le Système National des Données de Santé (SNDS) avec pour tâche " de contribuer à la recherche, aux études, à l'évaluation, et à l'innovation dans les domaines de la santé [...] » (12) en offrant le cadre et l'hébergement de milliards de données cliniques produites par ces structures. Par ailleurs, afin d'améliorer les soins, les établissements hospitaliers se regroupent en Groupements Hospitaliers de Territoire (GHT), et la mutualisation de leurs systèmes d'information prévue par la loi du 26 janvier 2016 a eu lieu le 1er janvier 2018. Elle sera exécutée 3 ans après par la convergence officielle de tous les systèmes d'information (13). Les données volumineuses provenant de différents hôpitaux vont donc constituer une richesse d'information médicale sans précédent, donnant la possibilité d'extraire un savoir ou une connaissance (14). Ces bases de données médicales, souvent hétérogènes, devront être adaptées à I'IA et d'accès fluidifié (10). Elles devront également être complétées par les données provenant de la médecine libérale (cliniques et cabinets de soignants) et du monde digital citoyen (informations issues des réseaux sociaux et requête Internet, capteurs esanté et objets connectés), ce qui conduira à l'accumulation de données massives (15). Le recueil des données de santé et psycho-sociales ne se fera donc plus uniquement par les professionnels de santé mais également à travers un ensemble de capteurs intégrés à l'individu. L'allergologie et les allergologues doivent donc s'y préparer.

L'IA ouvre ainsi de nouvelles possibilités pour innover en matière de recherche, de pratique clinique diagnostique et thérapeutique et de prévention. Elle permet de soumettre des hypothèses diagnostiques, d'analyser automatiquement et d'exploiter les résultats d'examens complémentaires (imagerie médicale notamment), de faire des propositions thérapeutiques pertinentes, personnalisées, de les suivre et donc à termes de proposer et d'évaluer les politiques de santé publique.

\section{Plateforme intelligente de gestion des cas complexes}

La solution AdviceMedica est basée sur un constat très simple : (i) on nous enseigne à la faculté le cas moyen or en pratique tous nos cas dévient de ce cas moyen pédagogique; (ii) en fonction de notre capacité de mémorisation, de notre expérience, il est des cas pour lesquels nous avons besoin de conseils, d'avis; (iii) les médecins ont l'habitude de s'entraider dans la gestion des cas inhabituels; (iv) la non résolution des cas complexes est source d'erreurs médicales. 
Lorsque les RCP n'existent pas (c'est-à-dire la plupart des maladies non cancéreuses) ou ne sont pas assez précises, que le praticien a peu d'expérience (début de carrière, maladies peu fréquentes ou extrêmement hétérogènes) ou est réfractaire aux RPC ou encore est "isolé" (territoires d'outre-mer, Afrique, médecins avec vacations hospitalières ne pouvant pas s'appuyer sur des services avec plusieurs confrères experts), la confrontation avec l'expérience de pairs et d'experts est fondamentale. Le contact direct n'est cependant pas évident, il est rarement instantané. C'est dans ce cadre précis que s'inscrit la plateforme collaborative AdviceMedica, utilisable par le médecin à son cabinet, et qui fait maintenant partie du quotidien de nombreux allergologues francophones. Un expert pour AdviceMedica c'est un confrère qui a déjà été confronté à un cas similaire et apporte des éléments cruciaux de réponse.

AdviceMedica fournit depuis un an une solution de communication asynchrone en réseaux et permet à des médecins d'échanger sur les cas complexes qu'ils rencontrent. C'est une solution d'entre aide collaborative, de télé-expertise communautaire pour la résolution de cas difficiles faisant appel à la mémoire collective; ces cas résolus sont stockés et se ressemblent parfois. La recherche de similarités par fouille profonde de données (data mining) peut donc techniquement être automatisée grâce aux outils de l'IA.

AdviceMedica gère une dizaine de réseaux hyperspécialisés en allergologie, dont deux d'entre eux (insectes piqueurs, Revidal) existaient antérieurement et utilisent maintenant la plateforme. Certains réseaux sont privés permettant aux membres de groupes de travail d'échanger sans diffusion de leurs réflexions. Mais la majorité des réseaux sont destinés à l'entraide collaborative entre médecins. En pratique, un médecin désireux de partager un cas et de poser une question à ses collègues, envoie une description de son cas à l'adresse de son réseau (ex. reseauinsectes.piqueurs@advicemedica.com). L'inscription à la plateforme est gratuite. Le email est alors géré par la plateforme AdviceMedica et renvoyé à l'ensemble des membres de ce réseau qui répondent, s'ils le souhaitent, quand ils le souhaitent. Des remontées d'information sont possibles en interrogeant directement les réseaux, permettant en 2018 de participer au plan de contingence de l'ANSM faisant suite à la rupture des extraits de venins d'hyménoptères et à l'évaluation des cas incidents indésirables au slime (16).

Techniquement, AdviceMedica est une application web composée d'un front office et d'un back office. Ce dernier surveille l'activité d'une ou plusieurs adresses emails et réagit en fonction de scénarios prédéfinis à l'arrivée d'un nouveau message. Ces scénarios sont basés sur le contenu, l'émetteur, les dates et l'historique du message. Le paramétrage et le suivi sont accessibles depuis un navigateur internet pour les administrateurs de l'application. Les interfaces sont construites à partir de pages HTML augmentées par du code pour la présentation, les interactions et la navigation.

Plus de 1000 médecins allergologues participent à l'un et/ou l'autre de ces réseaux, 30 à 60 cas sont envoyés par mois et obtiennent en moyenne 3,4 réponses par cas en 4 à 72 heures. Les données (à ce jour près de 1500 cas résolus) sont anonymes et en conformité avec les règlements et les lignes directrices régissant la pratique médicale, le règlement général de protection des données et l'éthique en France et en Europe. Une charte d'utilisation est en cours de validation juridique, de nouveaux décrets ayant vu le jour dans le courant de l'année 2018. 
Un projet en cours, financé par la Société Française d'Allergologie nous permettra dans son objectif principal d'établir une mesure de similarité syntaxique entre documents décrivant des cas complexes par application de méthodes récentes de traitement du langage naturel $(17,18)$. Les objectifs secondaires de ce projet sont (i) d'établir des mesures de similarité plus spécifiques (19) entre des sous-ensembles des documents ne comprenant que des profils de patients ou des tests in vivo (tests cutanés, tests de provocation) ou in vitro (dosages biologiques) ; (ii) d'extraire des relations afin de qualifier le lien sémantique entre entités médicales $(20,21)$. Un objectif exploratoire nous permettra de déduire des similarités précédentes et des relations sémantiques identifiées des corrélations et tendances dans les cas complexes en allergologie.

Le traitement automatique du langage naturel (natural language processing) et l'apprentissage automatique (machine learning) sont aujourd'hui les moyens de réaliser ces mesures de similarité, et sont l'objet de ce projet de recherche en IA, appliqué à l'allergologie et aux échanges entre médecins d'avis sur des cas complexes.

Ces travaux devraient nous permettre de :

- Grouper les cas de la base avec les différentes mesures de similarité ;

- Proposer automatiquement des cas similaires au médecin interrogateur ;

- Utiliser les relations sémantiques entre entités médicales ;

- Assurer une veille sanitaire automatique (répétition d'occurrences) ;

- Analyser plus finement et communiquer des séries de cas similaires et originaux, en accord avec les médecins participants (charte).

Le produit de cette recherche appliquée sera immédiatement transposable en pratique puisque le code de similarité sera déposé sur la plateforme et activé. L'allergologie sera alors la première spécialité à disposer d'un réseau d'entre aide collaborative, renforcé par des analyses automatiques à la pointe de l'IA.

\section{Remerciements}

Les auteurs remercient chaleureusement la Société Française d'Allergologie pour l'obtention d'une bourse de recherche 2019.

\section{Déclaration de liens d'intérêt}

Pascal Demoly a reçu des honoraires de conseil des laboratoires ALK, Stallergenes Greer, Allergy Therapeutics, YSlab, Sanofi, Bausch \& Lomb, AstraZeneca, Sanofi, ASIT Biotech et ThermoFisher Scientific; a reçu des honoraires pour des conférences sponsorisées par les laboratoires MYLAN, Chiesi, ALK, et Stallergenes Greer. Les auteurs envisagent de développer un projet entrepreneurial autour de cette idée AdviceMedica. 
Tableau 1 : Extrait du Code de Déontologie Médicale du Conseil National de l'Ordre des Médecins

Article 32 (article R.4127-32 du code de la santé publique)

Dès lors qu'il a accepté de répondre à une demande, le médecin s'engage à assurer personnellement au patient des soins consciencieux, dévoués et fondés sur les données acquises de la science, en faisant appel, s'il y a lieu, à l'aide de tiers compétents.

Article 33 (article R.4127-33 du code de la santé publique)

Le médecin doit toujours élaborer son diagnostic avec le plus grand soin, en y consacrant le temps nécessaire, en s'aidant dans toute la mesure du possible des méthodes scientifiques les mieux adaptées et, s'il y a lieu, de concours appropriés.

\section{Références}

[1] Schmidt HG, Norman GR, Boshuizen HP. A cognitive perspective on medical expertise: theory and implications. Acad Med 1990; 65: 611-21.

[2] Gosh AK. Understanding medical uncertainty: a primer for physicians. JAPI 2004; 52: 739-42.

[3] Fox RC. The Evolution of medical uncertainty. Milbank Mem Fund Q Health Soc 1980; 58: 1-49.

[4] Hippocrates. Aphorisms. In: Adam F. The genuine works of Hippocrates. London: The Syndenham Society; 1869: 697.

[5] Haynes RB, Devereaux PJ, Guyatt GH. Physicians' and patients' choices in evidence based practice. BMJ. 2002; 324: 1350.

[6] Litaker D, Tomolo A, Liberatore V, Stange KC, Aron D. Using complexity theory to build interventions that improve health care delivery in primary care. J Gen Intern Med 2006; 21: S30-4.

[7] Thompson DS, Fazio X, Kustra E, Patrick L, Stanley D. Scoping review of complexity theory in health service research. BMC Health Serv Res 2016; 12: 87.

[8] Thibert C. Erreurs médicales : un recensement difficile. 23.11.2017. Disponible sur :

http://sante.lefigaro.fr/article/combien-d-erreurs-medicales-chaque-annee-en-france-/ (consulté le 27.08.2018).

[9] Castel P. La gestion de l'incertitude médicale : approche collective et contrôle latéral en cancérologie. Sciences Sociales et Santé 2008; 26: 9-32.

[10] Villani C. Donner un sens à l'intelligence artificielle. 2018. Disponible sur :

https://www.aiforhumanity.fr/pdfs/9782111457089_Rapport_Villani_accessible.pdf (consulté le 26.08.2018).

[11] Hollande F. Loi n 2016-41 du 26 janvier 2016 de modernisation de notre système de santé. Journal officiel, $n^{\circ} 0022,27$ janv. 2016. Disponible sur :

https://www.legifrance.gouv.fr/affichTexte.do?cidTexte=JORFTEXT000031912641\&categorieLien=id (consulté le 24.02.2018).

[12] CNIL. Protéger les données personnelles, accompagner l'innovation, préserver les libertés individuelles. SNDS. 2018. Disponible sur : https://www.cnil.fr/fr/snds-systeme-national-des-donnees-de-sante (consulté le 24.02.2018).

[13] Remize M. SI des hôpitaux : mutualisation et dossier patient. Archimag, Oct. 2017, n 308, p. 18-19. 
[14] Raghupati W, Raghupati V. Big data analytics in health care: promise and potentia. Health Information Science and System. 2014. Disponible sur : https://www.biomedcentral.com/track/pdf/10.1186/2047-2501-23?site=hissjournal.biomedcentral.com (consulté le 09.06.2018).

[15] Marcon C, Grosjean S, Mallowan M. Introduction : Méthodes et stratégies de gestion de l'information par les organisations : des " big data " aux « thick data ». Revue COSSI, 2018, $n^{\circ} 1$. Disponible sur : http://revuecossi.info/1054-numeros-de-revue/n-1-2018-big-data-thick-data (consulté le 9.06.2018).

[16] Exposition au Slime : données des centres antipoison et remontée d'alertes du Revidal-Gerda et du réseau Allergos. 2018. https://www.anses.fr/fr/system/files/PRES2018CPA15.pdf.

[17] Mikolov T, Sutskever I, Chen K, Corrado G, Dean J. Distributed representations of words and phrases and their compositionality. Neural Information Precessing Systems (NIPS) 2013. https://arxiv.org/pdf/1310.4546.pdf.

[18] Le $Q$, Mikolov T. Distributed representations of sentences and documents. International Conference on Machine Learning (ICML), Beijing, China, 2014. https://cs.stanford.edu/ quocle/paragraph_vector.pdf.

[19] Xu J, Xu B, Wang P, Zheng S, Tian G, Zhao J, Xu B. Self-taught convolutional neural networks for short text clustering. Neural Netw 2017; 88: 22-31.

[20] Jat S, Khandelwal S, Talukdar P. Improving distantly supervised relation extraction using word and entity based attention. 6th Workshop on Automated Knowledge Base Construction (AKBC) at NIPS 2017, Long Beach, CA, USA. https://arxiv.org/abs/1804.06987.

[21] Verga P, Strubell E, McCallum A. Simultaneously self-attending to all mentions for full-abstract biological relation extraction. North American Chapter of the Association for Computational Linguistics (NAACL) 2018. https://arxiv.org/abs/1802.10569. 